\title{
Dust in Hot Gas: Far Infrared Emission from Three Local Elliptical Galaxies
}

\author{
Pasquale Temi ${ }^{1,2}$, William G. Mathews ${ }^{3}$, Fabrizio Brighenti ${ }^{3,4}$, Jesse D. Bregman ${ }^{1}$
}

\begin{abstract}
We present far-IR ISO observations of three early-type galaxies in the Virgo cluster. The data were recorded using the ISOPHOT instrument in both the P32 oversampled maps and the P39/37 sparse maps. The maps reach the limiting sensitivity of the ISOPHOT instrument at 60,90 , and $180 \mu \mathrm{m}$. Two of the most prominent elliptical galaxies in Virgo - NGC 4472 and NGC 4649 - clearly show no emission at far-IR wavelengths at a level of few tens of mJy, but NGC 4636 is detected at all three wavelengths. We have computed the far-IR emission from dust for NGC4472 and NGC4636 under the assumption that dusty outflows from evolving red giant stars are continuously supplying dust to the interstellar medium and that the grains, once diffused into the interstellar medium, are sputtered away by collisions with ions. While the calculated fluxes are consistent with the observed upper limits for NGC4472, the dust emission detected in NGC4636 support the hypothesis that additional dust has been accreted in a very recent ( $\lesssim$ few $10^{8}$ yrs) merger with a dusty, gas-rich galaxy.
\end{abstract}

Subject headings: galaxies: elliptical and lenticular; galaxies: ISM; galaxies, individual(NGC 4472, NGC 4636, NGC 4649); infrared: galaxies; infrared: ISM

\section{Introduction}

The interstellar medium (ISM) in elliptical galaxies, although mostly X-ray emitting hot gas, contains a significant amount of dust and cold gas of uncertain origin. Most of the ISM within the half-light radius is produced by stellar mass loss. Since the mean stellar

\footnotetext{
${ }^{1}$ Astrophysics Branch, NASA/Ames Research Center, MS 245-6, Moffett Field, CA 94035.

${ }^{2}$ SETI Institute, Mountain View, CA 94043.

${ }^{3}$ University of California Observatories/Lick Observatory, Board of Studies in Astronomy and Astrophysics, University of California, Santa Cruz, CA 95064.

${ }^{4}$ Dipartimento di Astronomia, Università di Bologna, via Ranzani 1, Bologna 40127, Italy.
} 
abundance in typical giant elliptical galaxies is comparable to solar, envelopes ejected from evolving giant stars should produce dust just as observed in similar stars in the Milky Way. In fact, relatively hot circumstellar dust has been observed in many elliptical galaxies at $\sim 10 \mu \mathrm{m}$ with both IRAS and ISO satellites (Knapp et al. 1989; Ferrari et al. 2002; Athey et al. 2002). This mid-IR emission has a de Vaucouleurs profile similar to the background starlight and is therefore consistent with circumstellar dust recently ejected from evolving red giant stars. After about $10^{4}-10^{5}$ years, the gas and dust ejected by giant stars orbiting in elliptical galaxies expands sufficiently to interact with the ambient hot gas. According to currently popular assumptions, as the ejected gas is dynamically disrupted, it is (shocked and conductively) heated to the virial temperature $\left(\sim 10^{7} \mathrm{~K}\right)$ of the hot ISM and the new dusty gas merges with its hot environment. When surrounded by gas at $\sim 10^{7} \mathrm{~K}$, the dust is sputtered until it also merges into the hot gas typically on a longer timescale. However, during the sputtering time, which varies with galactic radius, the dust receives enough energy from electrons and starlight to emit copiously in the mid and far infrared.

A substantial number of elliptical galaxies have been observed using the ISOPHOT (Lemke et al. 1996) instrument on board the ISO (Kessler et al. 1996) satellite. The spectral region covered by ISOPHOT, between 60 and $200 \mu \mathrm{m}$, together with its mapping capabilities and good angular resolution, make the instrument well suited for detecting thermal emission from interstellar dust. In particular the extension of its spectral coverage to longer wavelengths, relative to the IRAS satellite, provides a better sampling of the far-infrared emission where the expected flux density from the cold interstellar dust component peaks. ISOPHOT also has a fainter limiting magnitude compared to IRAS and diffraction limited imaging that could help resolve and disentangle the various expected sources of emission: central dust clouds, circumstellar dust, and truly interstellar dust distributed throughout the galactic volume. Here we report on ISOPHOT observations and analyses of three bright nearby early-type galaxies that are very similar in their optical parameters but are significantly different in their far-IR emission.

\section{Observations and Data Reduction}

ISOPHOT observations of early-type galaxies have been taken in several observing modes (Astronomical Observing Template, referred to as AOTs), including the oversampled maps P32, the P37/38/39 sparse maps and P22 multi-filter photometry data. Here we present data from three very bright ellipticals located in the Virgo cluster: NGC4636, NGC4649 and NGC4472.

NGC4636 has been observed using the C100 $3 \times 3$ and the C200 $2 \times 2$ detector arrays with the sparse map on-off mode (AOT P37/39) at 60 and $100 \mu \mathrm{m}(\mathrm{C} 100)$ and at $180 \mu \mathrm{m}(\mathrm{C} 200)$. 
Observations were made in each filter with one on-source single point staring mode and one off-source exposure. The background position was located $\sim 12^{\prime}$ north of the target, on a blank sky position. Data reduction and calibration were carried out using the PHT Interactive Analysis tool PIA, version 9.1 (Gabriel et al. 1997) together with the calibration data set V4.0 (Laureijs et al. 1998). The reduction included correction for the non-linear response of the detectors, readout deglitching, and linear fitting of the signal ramps. After resetting of all ramp slopes and subtracting the dark current, the calibration of detector responsivity and its changes was performed using measurements of the thermal fine calibration source (FCS) on board. The sky subtracted on source signal was then corrected for the fraction of the point-spread function included within the field of view of the detectors. Finally, the flux densities were extracted applying a color correction assuming that the SED of the galaxy can be approximated with a blackbody of temperature $30 \mathrm{~K}$. The statistical errors derived from signal processing are about 5-20\%, depending on the wavelength range and object brightness, but systematic errors due to absolute calibration accuracy are estimated to be $30 \%$ (Klaas et al. 1998). The derived flux density for NGC4636 in the three filters is shown in Table 1. Values are in good agreement with previous IRAS observations.

NGC4649 and NGC4472 have been observed in the P32 mode in three broadband filters at 60, 90 and $180 \mu \mathrm{m}$ using the $\mathrm{C} 100$ and C200 detectors. The maps were obtained scanning the spacecraft $\mathrm{Y}$ and $\mathrm{Z}$ axes in a grid of $4 \times 4$ (NGC4649) and $5 \times 4$ (NGC4472) points. The focal plane chopper was stepped at intervals of one-third of the detector pixel at each raster position, providing a sky sampling in the Y direction of $15^{\prime \prime}$ and 30" for the C100 and C200 detectors. All the maps were centered on the position of the nuclei of the two galaxies covering a field of about $9^{\prime} \times 6^{\prime}$. To reduce the P32 data we used a dedicated software package (P32TOOLS) developed at MPI Kernphysik in Heidelberg, and supported by the VILSPA ISO Data Center (Tuffs \& Gabriel 2002). The new routines allow a proper correction for transients in PHT32 measurements. The ISOPHOT C200 and especially the C100 detectors have a complex non-linear response as a function of illumination history on time scales of 0.1-100 sec, depending on the absolute flux level as well as the flux changes involved. Since no theory exists to predict the detector response behavior, an empirical model has been found to apply the transient correction to the data. The severity of the problem depends on both the source/background ratio and the dwell time at each pointing direction. Generally, ISOPHOT data are reduced in one pass from the raw input data with full time resolution to the final calibrated map. To correct for responsivity drift effects, however, it is necessary to iterate between a sky map and the input data at full time resolution. This different concept has been implemented in the dedicated data reduction package. The implementation of these new algorithms has been already successfully applied to an extended sample of Virgo cluster galaxies by Popescu et al. (2002). Great effort has been devoted to the removal of spikes and longer lived tails in the detector responsivity that are often generated by cosmic 
rays hitting the detector pixels. For the observations presented here, which are faint discrete sources, an accurate deglitching procedure becomes critical in determining the instrumental sensitivity. Although P32TOOLS applies specific deglitching procedures to remove glitches from the data, some residuals are still present in the maps made with the $\mathrm{C} 100$ detector. Removal of these artifacts was fully achieved for the C200 detector.

The photometric calibrations of discrete sources performed with the ISO flux scale have been tested against integrated flux densities measured by IRAS at 60 and $100 \mu \mathrm{m}$ bands on Virgo cluster galaxies (Tuffs et al. 2002). A very good linear correlation is found between the ISO and IRAS fluxes [see Figures $7 a$ and $7 b$ in Tuffs et al. (2002)].

Elliptical galaxies NGC4472 and NGC4649 were not detected at any of the three wavelengths observed by ISOPHOT $(60,90$, and $180 \mu \mathrm{m})$. Figure 1 shows the $90 \mu \mathrm{m}$ maps for these two

galaxies; the bright source at the edge of the NGC4649 map is its spiral companion NGC4647 and it is prominent at all three wavelengths. The angular resolution of the oversampled maps clearly resolves NGC4647 showing that there is no contribution to the signal associated with NGC4649. In the literature, IRAS detections at 60 and $100 \mu \mathrm{m}$ have been erroneously attributed to NGC4649, simply because the spatial resolution of the IRAS observations, $3^{\prime}$ $5^{\prime}$ at $100 \mu \mathrm{m}$, is very large and comparable to the optical size of most elliptical galaxies. The maps reach the limiting sensitivity of the ISOPHOT instrument at a level of a few tens of mJy and the $3 \sigma$ upper limits are presented in Table 1.

\section{A MODEL FOR NGC 4472 AND NGC 4636}

We estimate the FIR emission from dust dispersed in the ISM along the lines of Tsai \& Mathews $(1995,1996)$. A full description of the method will be presented elsewhere (Temi et al. 2003) In brief, we assume that the grains are ejected from evolving stars and mix with the hot ISM in a short time, $\sim 10^{5}-10^{6}$ yr (Mathews 1990). Once diffused into the hot gas, the grains are sputtered at a rate

$$
\frac{d a}{d t}=-3.2 \times 10^{-14} n_{p}\left[1+\left(2 \times 10^{6} / T\right)^{2.5}\right]^{-1} \mu \mathrm{m} \mathrm{s}^{-1}
$$

where $a$ is the grain radius (in $\mu \mathrm{m}$ ) and $n_{p}$ is the proton density (Draine \& Salpeter 1979; Tsai \& Mathews 1995)

Let $N(r, a) d a$ be the steady state number of grains per $\mathrm{cm}^{3}$ with radius between $a$ and $a+d a$ at galactic radius $r$. Tsai \& Mathews (1995) showed that the grain population must satisfy

$$
\frac{\partial}{\partial a}\left[N\left(\frac{d a}{d t}\right)\right]=S(r, a)
$$


where $S(r, a) d a$ is the rate at which grains with radii $a$ are expelled from stars per $\mathrm{cm}^{3}$. Mid-IR $(6-15 \mu \mathrm{m})$ ISO observations of a sample of elliptical galaxies, including NGC4472, detect strong dust emission that can be understood as emission from circumstellar dust from mass-losing AGB stars at or near their globally expected rate (Athey et al. 2002). We assume $S(r, a)=S_{o}(r) a^{-s}$ for $0<a<a_{\max }$, with $s=3.5$ (Mathis et al. 1977), so the coefficient

$$
S_{o}(r)=\frac{3 \delta \alpha_{*} \rho_{*}}{4 \pi \rho_{g} 10^{-12}} \frac{(4-s)}{a_{\max }^{4-s}}
$$

depends on the rate that the old stellar population ejects mass $\alpha_{*}=4.7 \times 10^{-20} \mathrm{~s}^{-1}$, the stellar density $\rho_{*}(r)$, the initial dust to gas mass ratio scaled to the stellar metal abundance $\delta=(1 / 150) z(r)$, and the density of silicate grains, $\rho_{g}=3.3 \mathrm{gm} \mathrm{cm}^{-3}$. The solution of Equation (2) is then

$$
N(r, a)=\left|\frac{d a}{d t}\right|^{-1} \frac{S_{o}(r)}{(s-1)} a^{1-s} \quad a \leq a_{\max }
$$

The grain temperature is determined by the balance of heating (by both absorption of stellar radiation and by electron-grain collisions) and cooling:

$$
\int_{0}^{\infty} 4 \pi J_{*}(r, \lambda) Q_{a b s}(a, \lambda) \pi a^{2} d \lambda+4 \pi a^{2}(1 / 4) n_{e}\left\langle v_{e} E_{e}\right\rangle \tau(a)=4 \pi a^{2} \sigma_{S B} T_{d}(r, a)^{4}\left\langle Q_{a b s}\right\rangle\left(T_{d}, a\right) .
$$

Since grains experience a relatively high collision frequency with electrons and photons, we have not considered stochastic temperature excursions of individual grains (Tsai \& Mathews 1995). Since the $9.7 \mu \mathrm{m}$ silicate feature is seen in emission in a number of elliptical galaxies, including NGC4472 (Athey et al. 2002), we assume that all grains have properties similar to astronomical silicates (Laor \& Draine 1993). Moreover, to simplify the calculations we approximate the absorption coefficient with $Q_{a b s} \approx a \psi(\lambda)$ and the Planck-averaged values with $\left\langle Q_{a b s}\right\rangle \approx 1.35 \times 10^{-5} T_{d}^{2} a$ [see Draine \& Lee (1984)]. The mean intensity of starlight is found by integrating over a de Vaucouleurs stellar profile modified with a core of slope $\rho_{*} \propto r^{-p}$ within $r_{c}$. At a distance $d=17 \mathrm{Mpc}$, NGC4472 has an effective radius $R_{e}=8.57$ kpc, total stellar mass $M_{*, t}=7.26 \times 10^{11} M_{\odot}$ [assuming $M_{*, t} / L_{B}=9.2$ (van der Marel 1991)], core radius $r_{c}=200 \mathrm{pc}$ and $p=0.9$ (Faber et al. 1997). For NGC 4636, assuming again $d=17 \mathrm{Mpc}, R_{e}=8.32 \mathrm{kpc}, M_{*, t}=3.80 \times 10^{11} M_{\odot}, M_{*, t} / L_{B}=10.74, r_{c}=260 \mathrm{pc}, p=1.0$. The collisional heating term in Equation (5) includes a correction $\tau(a)$ for small grains which do not completely stop the electrons (Dwek 1986) and $\left\langle v_{e} E_{e}\right\rangle=\sqrt{32 / \pi m_{e}}(k T)^{3 / 2}$ is an average over a Maxwellian distribution. The ISM electron density and temperature are calculated using the fitting formulae given in Brighenti \& Mathews (1997).

Finally the FIR emissivity is calculated from

$$
j(r, \lambda)=\frac{1}{4 \pi} \int_{0}^{a_{\max }} N(r, a) 4 \pi a^{2} 10^{-8} Q_{a b s}(a, \lambda) \times \pi B\left(T_{d}(r, a), \lambda\right) d a .
$$


Figure 2 shows the resulting dust temperature profile for NGC 4636, for grains of three different sizes. Slightly higher temperatures are predicted for NGC 4472. We find that collisional heating dominates over stellar radiation heating for grain sizes $a \leq 0.3 \mu \mathrm{m}$ and $a \leq 1 \mu \mathrm{m}$ for NGC 4472 and NGC 4636 respectively.

The computed far-IR spectra calculated for $a_{\max }=1 \mu \mathrm{m}$ and $a_{\max }=0.1 \mu \mathrm{m}$ are shown in Figure 3, together with the ISO detections or upper limits. For NGC 4472 the predicted fluxes are consistent with the ISO upper limits provided $a_{\max } \leq 1 \mu \mathrm{m}$. Thus, no strong constraints on the properties of interstellar dust can be derived for this galaxy. On the other hand, for NGC4636 the detected fluxes are a factor $\sim 50$ above the computed values at all three observed wavelengths, assuming $a_{\max }=1 \mu \mathrm{m}$. The shape of the calculated spectral energy distribution, however, fits the observations quite well, indicating that the excess emission is caused by a larger amount of dust rather than by hotter grains. The calculated flux ratios $F_{90 \mu \mathrm{m}} / F_{60 \mu \mathrm{m}}=2.7$ and $3.8\left(a_{\max }=0.1\right.$ and $\left.1 \mu \mathrm{m}\right) ; F_{180 \mu \mathrm{m}} / F_{90 \mu \mathrm{m}}=1.2$ and 2.1 $\left(a_{\max }=0.1\right.$ and $\left.1 \mu \mathrm{m}\right)$ compare reasonably well with the observed values $F_{90 \mu \mathrm{m}} / F_{60 \mu \mathrm{m}} \sim 2.6$ and $F_{180 \mu \mathrm{m}} / F_{90 \mu \mathrm{m}} \sim 1.6$. The model for NGC 4636 predicts a total mass of interstellar dust $M_{\text {dust }}=1.7 \times 10^{5} \mathrm{M}_{\odot}$ if $a_{\max }=1 \mu \mathrm{m}$ or $M_{\text {dust }}=1.7 \times 10^{4} \mathrm{M}_{\odot}$ for $a_{\max }=0.1 \mu \mathrm{m}$. We are led to speculate that NGC 4636 has accreted dust in a recent merger with a gas-rich galaxy. Other explanations appear less palatable. A higher (by a factor $\geq 50$ ) dust production rate by a younger stellar population would be inconsistent with the optical colors of NGC4636. Moreover, we found that the underluminosity of internally produced dust with respect to the observed values cannot be eliminated by varying the grain size distribution parameters $a_{\max }$ and $s$.

\section{Conclusions}

We have presented ISO observations of three bright elliptical galaxies in the Virgo cluster. While NGC 4472 and NGC 4649 emit no far-IR emission at the limiting sensitivity of the instrument, NGC 4636 is clearly detected. These observations have been interpreted with theoretical models for the far-IR emission from interstellar dust under the assumption that the circumstellar dust observed in the mid-IR (Athey et al. 2002) ultimately becomes interstellar and is exposed to the mean galactic starlight and to bombardment by thermal electrons in the hot gas. Grains receive somewhat more energy from electrons than from stellar photons. The calculated far-IR spectrum peaks at $\lambda \sim 180 \mu \mathrm{m}$, a spectral region not accessible to IRAS, but well covered by ISO.

Although NGC 4472 and NGC 4636 are similar in terms of mass, luminosity, hot gas content, and other physical parameters, they show a remarkable discrepancy in their far-IR emission. 
While the upper limits for NGC 4472 are consistent with emission from dust produced by the evolving stellar population, the model for NGC 4636 falls short by $\sim 50$ in explaining the observed fluxes. If the dust mixture would include a significant amount of graphite grains, the FIR emission would be at most a factor of few larger (Tsai \& Mathews 1996), still much too low to explain the observations. This strongly suggests that dust has been accreted by NGC4636 in a recent merging event with a gas-rich galaxy. A dust mass larger by a factor of about 50-70 is needed in order to match the observed far-IR luminosity, if its relative spatial distribution is similar to the one calculated. If the required extra-dust has an external origin, however, its distribution may be irregular and patchy. Without spatial information on the FIR emission we are not in the position to constrain the spatial location of the external dust. Ravindranath et al. (2001) find no compelling evidence for dust extinction in NGC4636 but the morphological properties of its stellar core are deviant. An asymmetrical distribution of diffuse $\mathrm{H} \alpha+[\mathrm{NII}]$ emission with an irregular velocity field has been detected by Caon, Macchetto \& Pastoriza (2001) in the central region of NGC 4636. The very disturbed hot ISM (Jones et al. 2002) also suggests a recent merging event and this is further supported by the apparent failure of the hot gas within $\sim R_{e} / 2=4 \mathrm{kpc}$ to be in hydrostatic equilibrium (Brighenti \& Mathews 1997).

The hypothetical merger must be very recent because of the short sputtering time. ¿From Equation (1) the sputtering time for grains of radius $a_{\mu}$ (in $\mathrm{m} \mu$ ) is $t_{\text {sputt }} \approx a_{\mu} /\left|d a_{\mu} / d t\right| \approx$ $1.2 \times 10^{6} a_{\mu} n_{e}^{-1}$ yrs. Since the gas density in NGC4636 varies as $n_{e} \approx 0.178 r_{k p c}^{-1.36} \mathrm{~cm}^{-3}$ (Brighenti \& Mathews 1997), we find $t_{\text {sputt }} \approx 1.2 \times 10^{8} a_{\mu}\left(r_{k p c} / R_{e, k p c}\right)^{1.36} \mathrm{yrs}$. The total mass of dust that merged is $\sim 10^{6}-10^{7} M_{\odot}$ and the associated gas would have mass $\sim 10^{8}-10^{9}$ $M_{\odot}$. For this gas to have been heated to $\sim 10^{7} \mathrm{~K}$ and for some of the dust to have survived, the dusty merger must have occurred less than $t_{\text {sputt }}$, or a few $10^{8}$ yrs ago. The total mass of hot gas in NGC4636 within $\sim R_{e} / 2$ where hydrostatic equilibrium fails, $\sim 2 \times 10^{8} M_{\odot}$, is comparable to that of the added gas, so considerable dynamical disruption is possible. Hydrostatic equilibrium would be reestablished in a few dynamical times which is $\sim 3 \times 10^{7}$ yrs at $r \sim R_{e} / 2=4 \mathrm{kpc}$. Dust survival and continued dynamical activity both indicate that an important merger may have occurred within the last few $10^{8} \mathrm{yrs}$. It would be interesting to seek evidence for the merged stars in the stellar spectrum of NGC4636.

This study is supported by NASA grant 399-20-01 for which we are very grateful. WGM is supported by grants from the NSF and NASA.

\section{REFERENCES}

Athey, A., Bregman, J. N., Bregman, J. D., Temi, P. \& Sauvage, M. 2002, ApJ, 571, 272 
Brighenti, F. \& Mathews, W. G. 1997, ApJ, 486, L83

Caon, N., Macchetto, D., Pastoriza, M. 2001, ApJS, 127, 39

Draine, B. T. \& Lee H. M. 1984, ApJ, 285, 89

Draine, B. T. \& Salpeter, E. E. 1979, ApJ231, 77

Dwek, E. 1986, ApJ302, 363

Faber, S. M., et al. 1977, AJ, 114, 1771

Ferrari, F., Pastoriza, M. G., Macchetto, F. D., Bonatto, C., Panagia, N., and Sparks, W. B. 2002, A\&A, 389, 355

Gabriel, C., Acosta-Pulido, J., Heinrichsen, I., Morriss, H., \& Tai, W-M., A.S.P. Conference Series, Vol. 125, 1997, p. 108.

Jones, C., et al. 2002, ApJ, 567, L115

Kessler M. F., Steinz U. A., Anderegg M. E., et al., 1996, A\&A, 315, L27

Klaas, U., Laureijs, R. J., Radovich, M. and Schulz, B. 1998, ISOPHOT Calibration Accuracies, $\mathrm{SAI} / 98-092 / \mathrm{rp}$

Knapp, G. R., Guhathakurta, P., Kim, D-W., Jura, M. A. 1989, ApJS, 70, 329

Laor, A. \& Draine, B. T. 1993, ApJ, 402, 441

Laureijs, R. J., Klaas, U., Richards, P. J. and Schulz, B. 1998, ISOPHOT Data User Manual, $\mathrm{SAI} / 95-220 / \mathrm{Dc}$

Lemke D., Klaas U., Abolins J., et al., 1996, A\&A, 315, L64

Mathews, W. G. 1990, ApJ, 354, 468

Mathis, J. S., Rumpl, W., Nordsieck, K. H. 1977, ApJ, 217, 425

Popescu, C. C., Tuffs, R. J., Volk, H. J., Heinrich, J., Pierini, D., Madore, D. F. 2002, ApJ, 567,221

Ravindranath, S., Ho, L. C., Peng, C. Y., Filippenko, A. V., \& Sargent, W. L. W. 2001, ApJ 122,653

Temi, P., Brighenti, F., Mathews, W. G. 2002, in preparation 
Tsai, J. C. \& Mathews, W. G. 1995, ApJ, 448, 84

Tsai, J. C. \& Mathews, W. G. 1996, ApJ, 468, 541

Tuffs, R. J., Popescu, C. C., Pierini, D., Volk, H. J., 2002, ApJS, 139, 37

Tuffs, R. J., \& Gabriel, C., 2002, In Press.

van der Marel, R. P., 1991, MNRAS, 253, 710 
Fig. 1.- Optical image (contours) superimposed to ISOPHOT $90 \mu \mathrm{m}$ map for NGC4649 (left panel) and NGC4472 (right panel). Both galaxies have not been detected at 60, 90, and $100 \mu \mathrm{m}$. The bright source at the edge of NGC4649 map is its spiral companion NGC4647.

Fig. 2.- Dust temperature for NGC4636 due to starlight and electron-grain collisions computed as a function of radial distance. For grains with size $a \leq 0.3 \mu \mathrm{m}$ the collisional heating is larger than radiative heating.

Fig. 3.- Computed Far-IR flux from NGC4636 and NGC4472 calculated for $a_{\max }=1 \mu \mathrm{m}$ (solid lines) and $a_{\max }=0.1 \mu \mathrm{m}$ (dashed lines). The ISO detections are shown as open triangles for NGC4472 (upper limits), and open circles for NGC4636. 


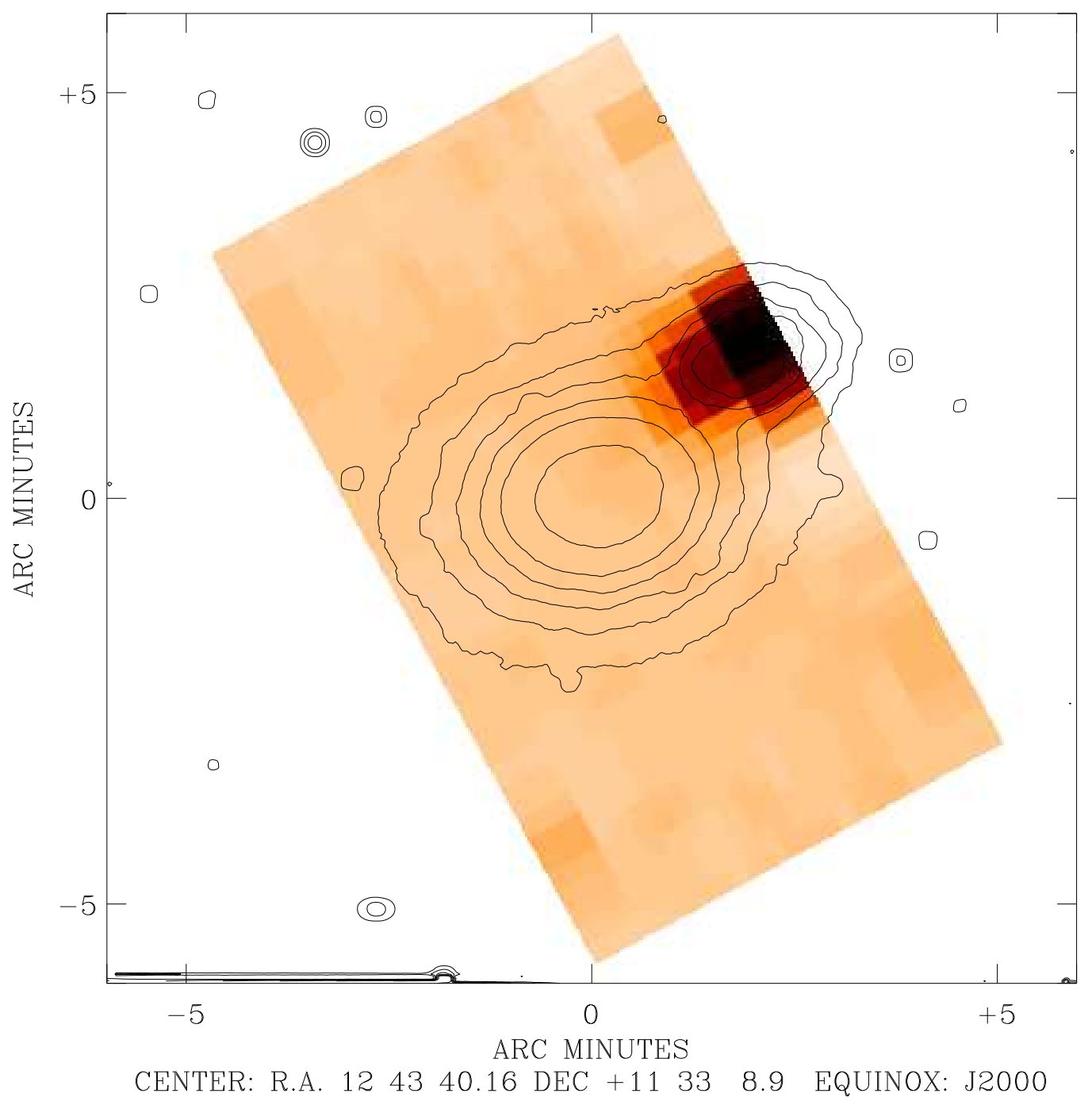




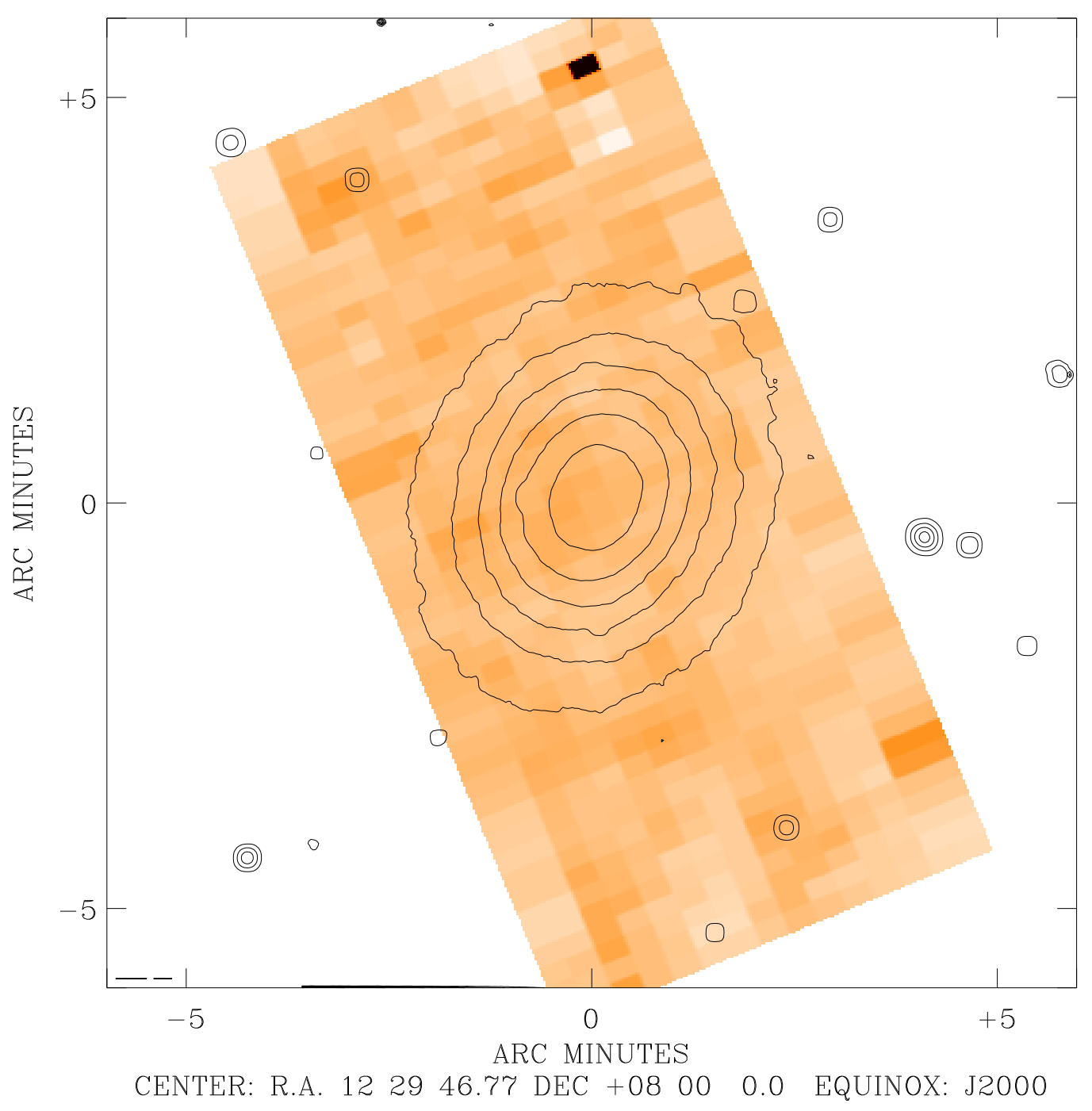




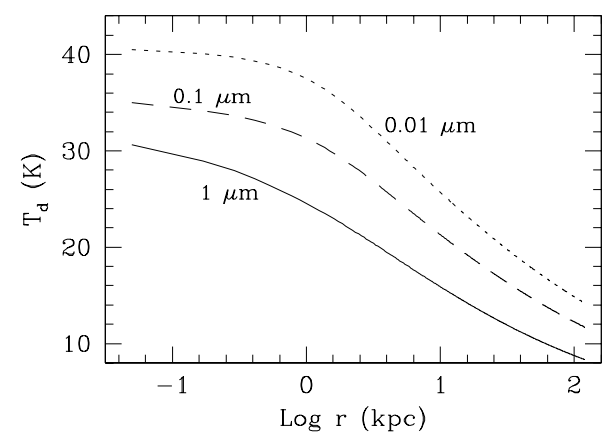




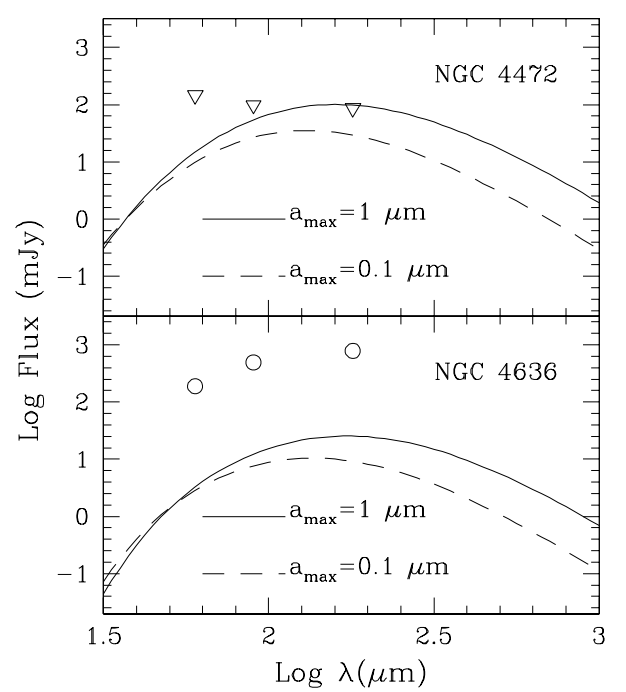


Table 1. ISOPHOT photometry.

\begin{tabular}{|c|c|c|c|c|c|}
\hline Galaxy & $\begin{array}{c}\log \left(\mathrm{L}_{\mathrm{B}}\right) \\
\left(\mathrm{L}_{B \odot}\right)\end{array}$ & $\begin{array}{l}\log \left(\mathrm{L}_{\mathrm{x}}\right) \\
\left(\operatorname{erg~s}^{-1}\right)\end{array}$ & $\begin{array}{c}\lambda \\
(\mu \mathrm{m})\end{array}$ & $\begin{array}{c}\text { Flux (ISO) } \\
\text { (mJy) }\end{array}$ & $\begin{array}{c}\text { Flux (IRAS) } \\
\quad(\mathrm{mJy})\end{array}$ \\
\hline \multirow[t]{4}{*}{ NGC $4649^{\mathrm{a}}$} & 10.73 & 41.21 & 60 & $\leq 137^{\mathrm{c}}$ & $900 \pm 65^{\mathrm{d}}$ \\
\hline & & & 90 & $\leq 85^{\mathrm{c}}$ & \\
\hline & & & 100 & $\ldots$ & $2310 \pm 270^{\mathrm{d}}$ \\
\hline & & & 180 & $\leq 110^{\mathrm{c}}$ & $\ldots$ \\
\hline \multirow[t]{4}{*}{$\mathrm{NGC} 4472^{\mathrm{a}}$} & 10.90 & 41.66 & 60 & $\leq 147^{\mathrm{c}}$ & $0 \pm 66$ \\
\hline & & & 90 & $\leq 99^{\mathrm{c}}$ & $\ldots$ \\
\hline & & & 100 & $\ldots$ & $0 \pm 94$ \\
\hline & & & 180 & $\leq 87^{\mathrm{c}}$ & $\ldots$ \\
\hline \multirow[t]{4}{*}{ NGC $4636^{\mathrm{b}}$} & 10.55 & 41.58 & 60 & $187 \pm 57$ & $200 \pm 34$ \\
\hline & & & 90 & $491 \pm 64$ & $\ldots$ \\
\hline & & & 100 & $\ldots$ & $560 \pm 179$ \\
\hline & & & 180 & $790 \pm 71$ & $\ldots$ \\
\hline
\end{tabular}

${ }^{a}$ Observed in the P32 oversampled map mode

${ }^{\mathrm{b}}$ Observed in P37/39 one position sparse map mode

${ }^{\mathrm{c}}$ No detection, $3 \sigma$ upper limit flux

${ }^{\mathrm{d}}$ The aperture photometry includes the spiral companion NGC4647 